
cultures

Les cahiers de l'Acedle

10-1 | 2013

Apprendre les langues autrement

\title{
Tensions épistémologiques en intercompréhension
}

\section{Christian Ollivier}

\section{OpenEdition}

Journals

Édition électronique

URL : http://journals.openedition.org/rdlc/1485

DOI : $10.4000 /$ rdlc. 1485

ISSN : 1958-5772

Éditeur

ACEDLE

Référence électronique

Christian Ollivier, "Tensions épistémologiques en intercompréhension », Recherches en didactique des langues et des cultures [En ligne], 10-1 | 2013, mis en ligne le 07 janvier 2013, consulté le 01 mai 2019. URL : http://journals.openedition.org/rdlc/1485 ; DOI : 10.4000/rdlc. 1485

Ce document a été généré automatiquement le 1 mai 2019.

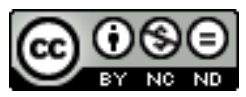

Recherches en didactique des langues et des cultures is licensed under a Creative Commons AttributionNonCommercial-NoDerivatives 4.0 International License 


\title{
Tensions épistémologiques en intercompréhension
}

\author{
Christian Ollivier
}

\section{Introduction}

1 L'intercompréhension, une des quatre approches plurielles retenues par le cadre de référence pour les approches plurielles des langues et des cultures (Carap) (Candelier et al., 2007) est, dans le domaine de la didactique des langues, un concept relativement jeune puisqu'il s'est répandu dans les années 1990 grâce notamment aux projets Galatea et EuRom4 en France et EuroComRom en Allemagne. La recherche en intercompréhension a d'abord été menée au sein de ses projets spécifiques et d'autres qui ont vu le jour au fil des années. Ce n'est que depuis quelques années que des liens plus étroits ont été tissés entre les projets et leurs équipes scientifiques. L'intercompréhension a ainsi réussi ces dernières années à se développer et à se structurer obtenant ainsi une visibilité et une reconnaissance de plus en plus forte. Des colloques ont été organisés auxquels ont participé des chercheurs venus de projets différents: en 2007 à Lisbonne, en 2011 à Rome et à Augsburg, finalement, en 2012 à Grenoble. Un réseau européen de l'intercompréhension (Redinter) a vu le jour en 2008 regroupant des chercheurs venus de projets et horizons différents. Une revue a été fondée par ce même réseau : Redinter-Intercompreensão.

Paradoxalement, le rapprochement des chercheurs a fait ressortir avec une acuité renforcée que, derrière le caractère fédérateur du terme d'intercompréhension, des différences de conceptions existent chez les spécialistes. M.-C. Jamet et D. Spita $(2010: 10)$ soulignent ainsi que,

à plusieurs reprises, lors des rencontres générales du Réseau Redinter, regroupant

les spécialistes européens d'intercompréhension, nous avons eu la perception que nous ne mettions pas tous sous le même terme d'intercompréhension - qui pourtant nous unit - une signification unitaire.

3 Cet article entend faire le point sur les différentes conceptions de l'intercompréhension à travers une étude de définitions publiées des années 1990 à aujourd'hui. 


\section{Travail épistémologique en intercompréhension}

Le terme, issu de la linguistique - la première attestation date de 1913 dans un ouvrage de J. Ronjat $(1913)^{1}-$, a subi depuis son passage en didactique des langues des mutations importantes. Quelques chercheurs ont consacré des études aux difficultés définitoires de l'intercompréhension et aux divergences existant entre certaines façons de penser. C. Degache (2006) dédie une partie de son dossier d'habilitation à diriger des recherches à la question, un ouvrage collectif de 2009 (Araújo e Sá, Hidalgo Downing, Melo-Pfeifer, Séré \& Vela Delfa, 2009) regroupe quelques articles sur le sujet. Le numéro 1 de la revue RedinterIntercompreensão (Ollivier \& Ferrão Tavares, 2010), entièrement dédié à l'épistémologie de l'intercompréhension, s'intéresse à l'émergence, à la définition et aux mutations du concept.

Dans ces travaux, nombreux sont les scientifiques qui font ressortir la polymorphie de l'intercompréhension (Degache, 2006, 2009; Gueidão, Melo-Pfeifer \& Pinho, 2009 ; Séré, 2009), nombreux aussi sont ceux (Escudé, 2010 ; Tost Planet, 2009 pour n'en citer que deux) qui, malgré les travaux qui ont vu le jour ces dernières années, continuent à souligner l'importance de mener - aujourd'hui plus que jamais - un travail épistémologique et définitoire de fond.

Un début de travail sur les définitions publiées par les spécialistes du domaine a été mené au sein du projet Redinter. M.-C. Jamet et D. Spita ont lancé, au sein du groupe de recherche du réseau, une collecte de définitions et ont publié les résultats de leur étude dans le numéro 1 de la revue Redinter-Intercompreensão (Jamet \& Spita, 2010). Dans ce même numéro, C. Ollivier (2010) analyse des définitions recueillies par voie de questionnaire lors d'une rencontre du réseau. Dans un article de la même année, M.-C. Jamet (2010) s'intéresse notamment aux définitions données par les dictionnaires.

$7 \quad$ Le présent article entend compléter ce travail par une étude d'ampleur plus importante que les travaux précédents. Si M.-C. Jamet et D. Spita s'appuient sur des définitions données par 33 auteurs, la présente étude prend en considération 118 définitions publiées entre 1993 et 2012 par 80 auteurs différents. Notons avant de présenter notre étude en soi qu'elle a été réalisée en grande partie au sein d'un projet bilatéral Amadeus conduit en collaboration avec M. Strasser de l'université de Salzburg.

\section{Méthodologie}

\section{Constitution du corpus d'étude}

8 La base est constituée des définitions collectées au sein du réseau Redinter. Nous avons enrichi le corpus en y ajoutant des définitions trouvées dans des articles traitant d'épistémologie de l'intercompréhension (cf. supra), nous avons finalement procédé à une recherche assistée par ordinateur pour récolter des définitions qui n'avaient, alors, été ni répertoriées comme telles ni étudiées. Ainsi, une collection de 403 publications numériques sur l'intercompréhension a été analysée électroniquement. De même, nous avons lancé plusieurs recherches sur Internet afin de repérer des portions de textes proposant des définitions de l'intercompréhension. Cette recherche a été essentiellement effectuée en allemand, en anglais et en français, langues des premiers projets pour 
l'allemand et le français, langue de publication fréquente pour l'anglais. Ont été ajoutés des travaux dans d'autres langues telles que l'italien et le portugais.

Nous avons formulé, dans des moteurs de recherche sur la Toile et dans notre bibliothèque électronique, des recherches comprenant les expressions suivantes susceptibles d'introduire une définition :

- "l'intercompréhension est" (Castagne, 2007a; Chavagne, 2010 ; Chilmonczyk, 2002 ; Daff \& Akissi Boutin, 2010 ; Jamet, 2011 ; Klein \& Reissner, 2003 ; Ploquin, 2005 ; Shopov, 2009)

- "par intercompréhension, on entend / nous entendons" (Meißner, 2012; Meißner, Beckmann \& Schröder-Sura, 2009; Pinho \& Andrade, 2008; Santos, 2007; Tafel, 2009; Zeevaert \& Möller, 2011);

- "l'intercompréhension comme" (Araújo e Sá, de Carlo \& Melo-Pfeifer, 2010; Martins \& Bartolomeu, 2010 ; Melo, 2007);

- "+intercompréhension +désigne" (Andrade \& Araújo e Sá, 2005; APIC (Association pour la Promotion de l'InterCompréhension des langues), n.d.; Meißner, 2003, 2004a ; Tost Planet, 2005) ;

- "l'intercompréhension constitue" (Gajo, 2008);

- "intercompréhension" (Blanche-Benveniste, 1997 ; Wuilmart, 2006).

Nous avons aussi recherché des réponses à des questions du type: "qu'est-ce que l'intercompréhension?" ou "qu'entend-on par intercompréhension?" (Bär, 2009; Délégation générale à la langue française et aux langues de France (DGLFLF), 2006; Redinter, 2012; Tost Planet, 2010), de même que des constructions appositives ou similaires (Blanche-Benveniste \& Valli, 1997 ; Degache, 2003 ; European Commission, n.d. ; Santos \& Andrade, 2005).

Les 118 définitions se répartissent comme suit dans le temps :

Figure - Répartition des définitions dans le temps, entre 1993 et 2012

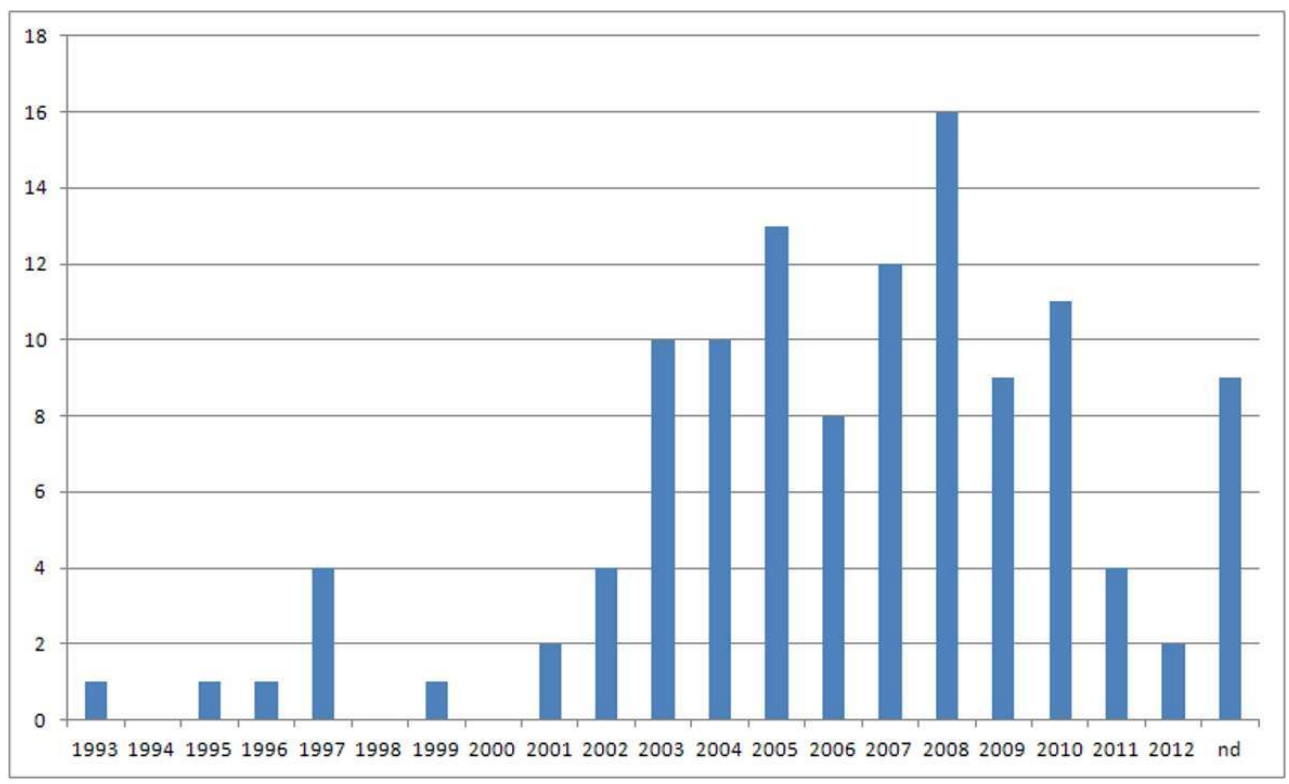

On note une présence faible de définitions dans les années 1990, due notamment au fait que, comme le rappelle M. Tost Planet (2005), plusieurs spécialistes emploient alors encore peu le terme, lui préférant des formulations différentes telles que "compréhension multilingue", "compréhension réciproque" ou bien encore "compréhension mutuelle". 
L'émergence, au début des années 2000, de nouveaux projets impliquant de nouveaux chercheurs s'intéressant à l'intercompréhension a conduit à faire augmenter le nombre de publications dans le domaine et ipso facto le nombre de définitions.

\section{Traitement des données}

13 Les définitions recueillies ont été entrées dans un tableur pour être codées à la suite d'une analyse de contenus puis traitées statistiquement. Le premier filtre appliqué est repris en partie aux analyses de M.-C. Jamet et D. Spita (2010), d'une part, et de C. Ollivier (2010), d'autre part. Il a permis de classer les définitions en trois catégories selon leur orientation praxéologique, didactique ou cognitive. Cette catégorisation découle, à l'origine, de l'étude du premier substantif apparaissant dans la définition et/ou de la description de cas précis d'intercompréhension dans les définitions.

Sous la rubrique praxéologique ont été réunies des définitions présentant l'intercompréhension comme une forme de communication :

l'intercompréhension fonctionnelle entre langues, c'est par exemple [...] un Français qui comprend un Allemand à Berlin ou qui renseigne un Italien à Paris, chacun parlant sa propre langue maternelle et comprenant la langue maternelle de l'autre (Castagne, 2007b : 19).

Ont été classées en didactique les définitions décrivant l'intercompréhension comme une approche de l'enseignement/apprentissage des langues :

IC as an educative and communicative goal, as a linguistic policy orientation and as a language learning and teacher training methodology, has been a domain of research from the earlier 1990ies onwards [...]. It is a specific approach to language learning and teaching, which aims to raise awareness, to develop the understanding of what we already know about the nature of language, communication systems and human interaction and to develop skills on all these domains. It is a pluralistic approach to languages and cultures [...] and it has various advantages. (Araújo e Sá et al., 2010: 281)

16 Finalement, nous avons considéré comme d'orientation cognitive, les définitions qui soulignent le fait que l'intercompréhension est une compétence, une capacité, etc.: " Interkomprehension ist die Fähigkeit, in einer Gruppe von Sprachen, die einen gemeinsamen Ursprung haben, kommunizieren zu können" (Klein \& Reissner, 2003 : 19).

L'étude de contenu a permis de faire ressortir des aspects récurrents dans les définitions qui ont fait l'objet d'une attention toute particulière. Il s'est avéré (cf. infra) que le filtre présenté ci-dessus n'apporte pas de résultats probants pour distinguer les différentes approches de l'intercompréhension. Au cours du travail d'analyse, la distinction entre les définitions qui conçoivent l'intercompréhension dans sa dimension réceptive et celles qui mettent en avant sa dimension interactive nous est apparue plus pertinente. Nous lui accorderons donc, dans cet article, une place de choix et aborderons succinctement deux autres aspects qui ont émergé de l'étude des définitions: la question des langues en présence et de leur apprentissage ou non. 


\section{Présentation et analyse des données}

\section{Conceptions praxéologique, didactique et cognitive de l'intercompréhension}

Historiquement, l'intercompréhension a d'abord été considérée comme une forme de communication. J. Ronjat (1913) considérait que si des individus s'intercomprenaient, c'est qu'ils parlaient des variétés différentes d'une même langue et que s'ils ne s'intercomprenaient pas, ils étaient des locuteurs de langues distinctes. Avec le passage en didactique des langues, le terme a parfois pris un sens didactique, l'intercompréhension devenant une approche de l'enseignement-apprentissage des langues. Ainsi, comme nous l'avons dit en entrée, le Carap (Candelier et al., 2007) la classe parmi les approches plurielles. On retrouve cette acception didactique très tôt chez L. Dabène (1995 : 106) qui conçoit l'intercompréhension comme un "apprentissage de la compréhension entre locuteurs de langue voisine". Avec cette vision didactique est apparue aussi une conception plus cognitive qui pose l'intercompréhension comme une compétence ou une capacité, tout au moins un processus cognitif de compréhension.

Dans les définitions étudiées, on note une répartition relativement équilibrée entre les conceptions praxéologiques (47) et cognitives (42) de l'intercompréhension et une position en retrait pour les définitions indiquant que l'intercompréhension est une approche didactique de l'enseignement/apprentissage des langues (34).

Il est aussi à noter que certaines définitions considèrent plusieurs dimensions du concept.

Ainsi F. Capucho évoque l'ancrage en didactique et une forme de communication avérée :

Dans ce sens, l'intercompréhension n'est pas une invention artificielle dans le champ de la didactique des langues, mais tout simplement la reconnaissance de processus naturels et spontanés mis en œuvre par des individus "non-savants" lors de contacts exolingues (Capucho, $2008: 239$ ).

Figure - Première catégorisation des définitions

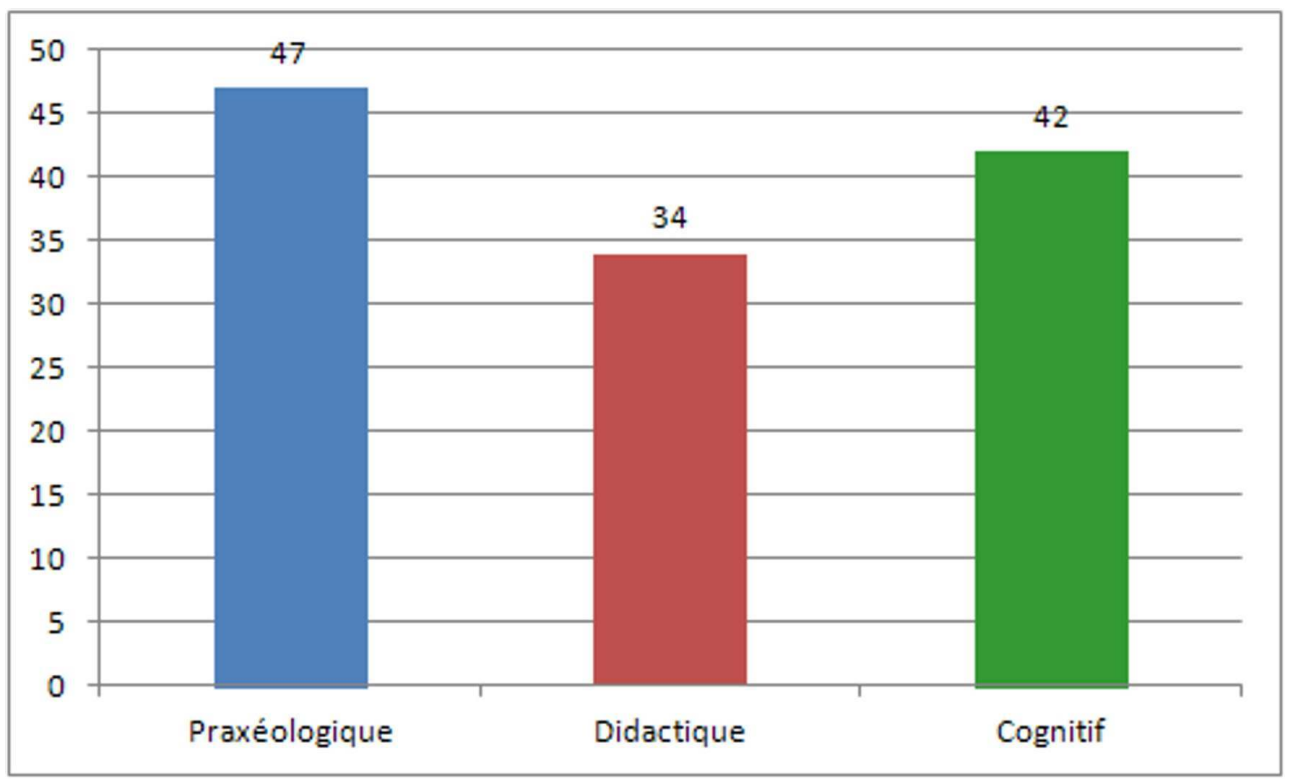


Comme le montrent des exemples de classement dans plusieurs catégories, les différences ne sont pas forcément antagonistes. Elles proviennent essentiellement de raccourcis de langage conduisant à la mise en relief d'un aspect du complexe intercompréhensionniste. $\mathrm{Au}$ départ, le terme d'intercompréhension sert à décrire un phénomène constatable dans la vie de tous les jours. Au passage en didactique des langues, ce qui est observable dans la réalité des échanges interhumains devient un objectif auquel s'intéresse la didactique de l'intercompréhension - souvent désignée alors sous le seul terme d'intercompréhension. Cette didactique vise alors le développement de compétences spécifiques ${ }^{2}$ qui permettent de mettre en œuvre la forme de communication spécifique qu'est l'intercompréhension. Une plus grande précision de langage dans le domaine, qui amènerait, par exemple, à parler dans certains cas de "didactique de l'intercompréhension" plutôt que d'intercompréhension, contribuerait à préciser l'angle d'approche de la notion.

Cette première catégorisation n'est pas fondamentalement celle qui fait ressortir les différences de conceptions les plus importantes. L'étude de la mise en avant dans les définitions de la réception ou de l'interaction fait ressortir des tensions épistémologiques nettement plus fortes, de même que des tensions entre théorie et pratique lorsque l'on se déplace sur le terrain de la didactique.

\section{Réception ou interaction}

23 L'une des spécificités de l'intercompréhension est que les chercheurs du domaine ont, dans le cadre de projets (essentiellement financés par la Commission européenne), conçu et réalisé des matériaux d'apprentissage parallèlement à leur travail de recherche. L'équipe de C. Blanche-Benveniste a ainsi publié la méthode EuRom4, l'équipe de L. Dabène a travaillé au projet Galatea, les Allemands ont développé les matériaux d'EuroComRom. En cela, l'intercompréhension est un domaine d'étude privilégié pour le lien épistémologique entre théorie et pratique. Nous allons montrer ici en quoi ce lien est parfois fait de tensions.

Dans le domaine de la didactique, les chercheurs en intercompréhension se sont d'abord intéressés à la réception écrite dans des langues génétiquement proches. Les projets Galatea, Eurom4 et EuroComRom visent explicitement l'apprentissage de la réception en langues romanes. Les matériaux didactiques proposés par ces projets sont fondés sur des textes écrits, il n'y a pas d'interaction plurilingue prévue pendant l'apprentissage. Plusieurs projets plus récents, incluant parfois des activités de réception orale, suivent ces orientations, qu'il s'agisse de projets liés aux pionniers tels que Eurom 5 ou EuroComGerm ou de projets indépendants comme Euro-mania, IGLO, Intercom, Eu+I (European Awareness and Intercomprehension), Itinéraires romans ou ICE (Intercompréhension européenne). Peu de projets prévoient de faire interagir les apprenants en langues différentes. Le projet qui a apporté la plus grande innovation en la matière est sûrement le projet Galanet sur la plateforme Internet duquel des apprenants en intercompréhension sont invités à échanger dans des langues romanes différentes pour réaliser une tâche commune. Dans cette nouvelle lignée, citons également le projet Intermar qui travaille en ce moment au développement d'activités pour les étudiants des académies maritimes européennes en proposant des interactions multilingues.

25 Si les projets privilégient la piste de la réception, les définitions considèrent, elles, l'intercompréhension nettement plus dans sa dimension interactive. 
Figure - Classement des définitions selon la compétence mise en avant

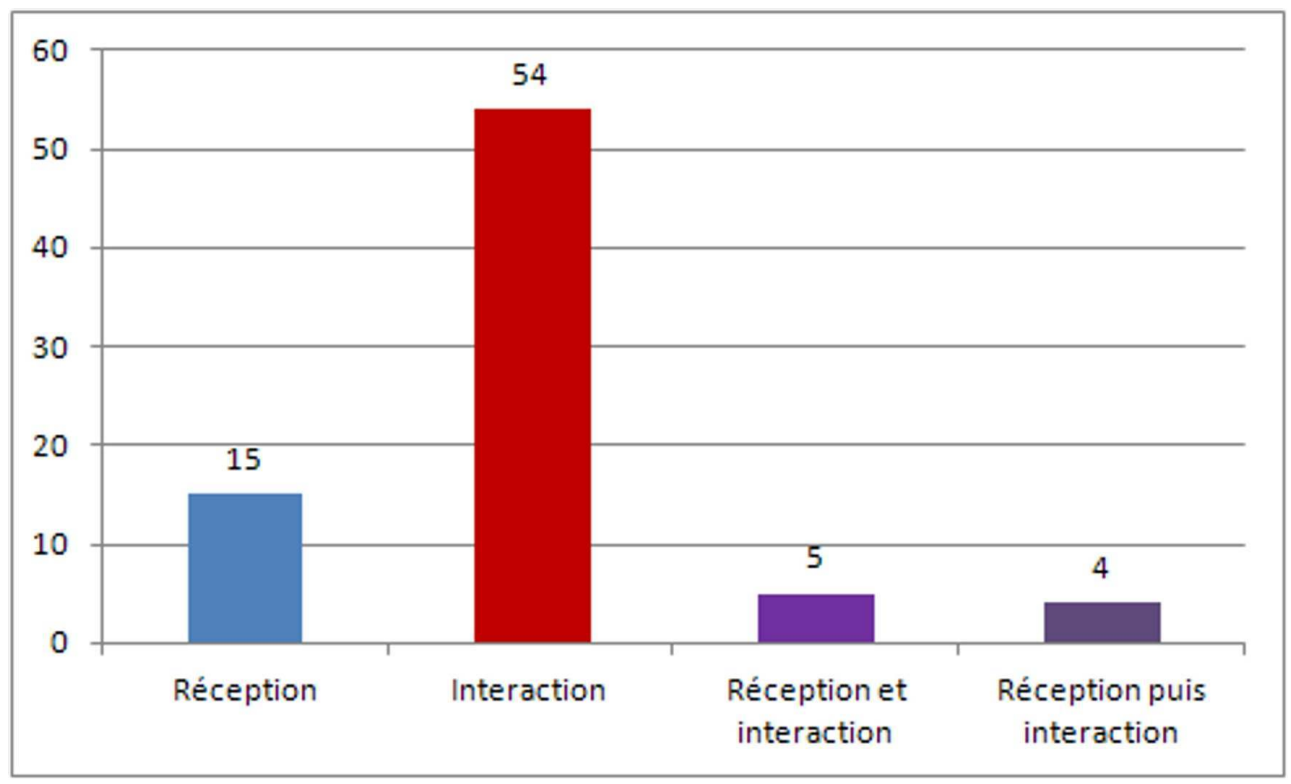

54 définitions évoquent l'intercompréhension comme interaction langagière. J.-C. Beacco et M. Byram donnent, à ce sujet, une définition explicite dans la préface à l'étude de référence publiée par P. Doyé (2005) :

On parle d'intercompréhension, par exemple, lorsque deux personnes communiquent, à l'oral ou par écrit, en s'exprimant dans des langues différentes, chacune comprenant la langue utilisée par l'autre (Beacco \& Byram, $2005: 6$ ).

Si l'on ne se sera pas surpris de trouver des définitions de ce type chez des chercheurs liés au projet Galanet (puis Galapro), on pourra cependant s'étonner de lire une vision interactive chez des chercheurs qui ont développé du matériel ne visant que la réception de l'écrit. C. Blanche-Benveniste et A. Valli (1997: 5) précisaient ainsi que l'intercompréhension, c'est "parler chacun sa langue et comprendre celle des autres" alors que le projet EuRom4 était exclusivement axé sur la réception de textes écrits. Certaines définitions apportent une possible explication, quelques chercheurs indiquent en effet que le développement d'une compétence de réception à l'écrit et à l'oral vise à terme une forme de communication entre locuteurs de langues différentes :

Die Fähigkeit zum Verstehen einer Sprache erstreckt sich dabei prinzipiell auf beide rezeptiven Fertigkeiten, nämlich das Lese- und das Hörverstehen [...] Interkomprehension verfolgt letzten Endes das Ziel einer Form der Kommunikation zwischen Mitgliedern einer Sprachenfamilie (Bär, 2009 : 24)

Mais nous n'avons jusqu'ici trouvé aucune trace de méthodologie ou de matériaux didactiques s'intéressant spécifiquement, par exemple, au passage de la réception de l'écrit à l'interaction orale. On note donc un décalage important entre des définitions très nombreuses qui considèrent l'intercompréhension dans sa dimension interactive et des matériaux didactiques qui, en majorité, visent le développement de la seule réception de l'écrit. Ceci peut s'expliquer en partie par le fait qu'en situation habituelle d'enseignement-apprentissage des langues, il n'est pas aisé de faire communiquer entre eux les apprenants dans des langues différentes, surtout si on se cantonne à des groupes de langues génétiquement apparentées, comme le font de nombreux projets (cf. infra). A cela s'ajoute probablement le fait que la réception écrite en langues génétiquement proches est la compétence la plus aisée à acquérir. Les intercompréhensionnistes mettent 
d'ailleurs souvent en avant le fait que leur approche de l'apprentissage des langues est caractérisée par une grande simplicité.

Le tournant vers des activités didactiques orientées vers l'interaction multilingue a été rendu possible par le développement des technologies de l'information et de la communication qui permettent de proposer des activités mettant en contact des locuteurs de langues différentes appelés à communiquer dans ces langues. Galanet exploite les potentialités d'Internet à travers une plateforme conçue spécialement pour le projet, Intermar va utiliser la plateforme d'apprentissage Moodle. Babelweb a développé un site de type web 2.0 pour permettre de faire cohabiter des publications en différentes langues romanes et promouvoir des interactions entre internautes s'exprimant dans des langues différentes. S'il existe des exemples de projets incluant des interactions multilingues, il est aussi important de rappeler que le nombre de ces projets reste encore très limité. Un autre décalage flagrant apparaît entre, d'une part, les conceptions de l'intercompréhension comme forme d'interaction et, d'autre part, les recherches faites dans le domaine et les matériaux didactiques produits. Jusqu'à présent, peu de chercheurs (Strasser, 2008) se sont vraiment préoccupés de la question de la production en situation d'intercompréhension. Pourtant, comme l'a souligné C. Ollivier (2012) lors du colloque "Intercompréhension compétences plurielles, corpus, intégration" qui s'est tenu à Grenoble en juin 2012, concevoir l'intercompréhension dans sa dimension interactive demande aussi de considérer la spécificité de ce type de production.

31 La recherche s'est probablement peu penchée sur la question parce qu'il n'est pas rare que l'on considère qu'en situation d'intercompréhension, les locuteurs utilisent chacun leur / une de leurs langue/s maternelle/s ou tout au moins une langue bien maittrisée (nous revenons sur cet aspect plus bas). On voit naître un début de recherche dans le domaine avec parfois même l'apparition du terme controversé d'"interproduction". Le colloque qui fera suite à celui qui s'est tenu à Grenoble en juin 2012 devrait accorder une place de choix à ces questions, comme l'ont annoncé les organisateurs lors de la séance de clôture.

Nous allons maintenant brièvement revenir sur la signification du préfixe "inter" à la lumière des focalisations sur la réception ou l'interaction.

\section{Le préfixe "inter"}

Selon la vision de l'intercompréhension, le suffixe "inter" prend des sens différents. Dans le cas de définitions qui restent dans le domaine du réceptif, l'intercompréhension est considérée comme la compréhension d'une langue fondée sur la connaissance d'autres langues, généralement génétiquement apparentées. On est alors dans de la compréhension sur la base de transfert interlinguistique, comme l'explique F.-J. Meißner dans deux des définitions étudiées: "generally, intercomprehension is the result of successful interlingual transferring" (Meißner, 2004b : 41s.) ou encore :

Unter ,Interkomprehension versteht allgemein man die Fähigkeit und den Vorgang, eine fremde sprachliche Varietät oder Sprache zu verstehen, ohne sie in ihrer natürlichen Umgebung erworben oder formal erlernt zu haben'.

Interkomprehension beruht auf Identifikationstransfer, das heißt darauf, dass man in ,fremden' Sprachen gefundene Formen (Wörter, Morpheme), Regularitäten und Funktionen (Aspekte, Tempora, Modi usw.) auf schon aus anderen Sprachen oder 
Varietäten bekannten Schemata zurückführen ... und verstehen kann (Meißner,

$2012: 234)$ langues a émergé. P. Doyé (2005) l'évoque explicitement dans son étude de référence pour le Conseil de l'Europe et quelques projets ont essayé de proposer des activités dépassant le cadre des langues apparentées. Eu+I est un projet pionnier dans ce domaine, ses langues cibles sont l'allemand, le bulgare, l'espagnol, le flamand, le français, le grec, l'italien, le portugais, le suédois et le turc. Ce type de projet met en avant des stratégies intralinguistiques surtout lorsqu'il s'agit de langues ne présentant que peu de similitudes avec les langues connues de l'apprenant. Le projet Intercom, qui propose des matériaux didactiques pour le portugais, l'allemand, le bulgare et le grec déclare ainsi "développer une didactique de l'intercompréhension fondée sur la mise en valeur des stratégies faisant appel aux aspects non-linguistiques de la compétence réceptive" ${ }^{13}$. Dans un tel cas, l'intercompréhension est un objectif second, l'apprentissage réceptif de langues visant à plus long terme l'intercompréhension au sens interactif. Ses projets sont donc plutôt situés dans le domaine de l'apprentissage réceptif en amont de l'intercompréhension.

\section{Perspectives en guise de conclusion} définitions et les projets : d'une part, on trouve les définitions qui mettent en avant la réception, sur la base de transferts interlinguistiques, de textes produits dans une langue "inconnue". 13 définitions évoquent le fait que ce type d'intercompréhension vise la réception dans une langue qui n'a pas été apprise que ce soit de façon formelle ou informelle. F.-J. Meißner et P. Doyé l'évoquent clairement dans leurs définitions de 2003 et 2005 : "Par le terme intercompréhension, on désigne la capacité de comprendre une langue étrangère sur la base d'une autre langue sans l'avoir apprise" (Meißner, 2003 : 31), "du point de vue de la compétence, l'intercompréhension est la capacité de comprendre d'autres langues sans les avoir apprises" (Doyé, 2005 : 7). En didactique, l'accent est alors mis sur l'acquisition de connaissances et de compétences de réception liées à l'utilisation des similarités et de régularités dans les différences existant entre langues génétiquement apparentées. 25 définitions sur les 118 étudiées évoquent explicitement la proximité génétique des langues en présence en situation d'intercompréhension. 

condition que les individus disposent d'un minimum de compétences permettant de mener des interactions exolingues impliquant la langue dans laquelle les autres partenaires de la communication s'expriment. Ainsi, rien n'empêche la communication de fonctionner si une personne s'exprime en japonais et l'autre en swahili dans la mesure où la première dispose de connaissances en swahili et la seconde en japonais. Il est important de noter que la langue d'expression n'est alors pas forcément la / une des langue/s maternelle/s de la personne, contrairement à ce que disent certaines définitions qui évoquent le fait que chaque interlocuteur s'exprime dans "sa" langue (25), précisant parfois qu'il s'agit de sa langue maternelle (10) ${ }^{4}$. Cette acception de l'intercompréhension suppose bien entendu un apprentissage tout au moins réceptif des langues en présence.

41 A cet apprentissage réceptif devrait, pensons-nous, s'ajouter un apprentissage de la production (en interaction) dans une situation d'intercompréhension qui envisage des stratégies d'adaptation du discours aux partenaires de la communication. Pour ne donner qu'un exemple extrêmement simple, dans de nombreuses situations d'intercompréhension, il sera préférable au niveau lexical de préférer le verbe transparent "téléphoner" au verbe "appeler". De même, une attention toute particulière devrait être portée aux questions interculturelles. Il nous semble en effet que les situations d'intercompréhension sont encore plus sensibles que d'autres puisque chacun s'exprime dans une langue différente, les langues utilisées pouvant ne pas appartenir aux langues maternelles des interlocuteurs. Ceci augmente encore le nombre de langues et de cultures en présence et demande une compétence plurilingue et pluriculturelle particulièrement développée.

Si la recherche et la didactique de l'intercompréhension comprise dans sa dimension réceptive (essentiellement à l'écrit) et impliquant des langues génétiquement apparentées est déjà largement développée, l'aspect visant le développement d'une compétence d'interaction multilingue laisse encore aux chercheurs de nombreuses questions à traiter en lien avec la notion de compétence plurilingue et pluriculturelle. 


\section{BIBLIOGRAPHIE}

\section{Bibliographie}

Andrade, A. I. \& Araújo e Sá, M. H. (2005). Educaçao em línguas em contexto escolar : da intervençao à reflexao. Aveiro : Universidade da Aveiro. http://www.ua.pt/cidtff/lale/Readobject.aspx? obj $=14139$

APIC (Association pour la Promotion de l'InterCompréhension des langues) (n.d.).

"L'intercompréhension, qu'est-ce que c'est ?" http://apic.onlc.fr/23-Quest-ce-que-cest.html

Araújo e Sá, M. H., de Carlo, M. \& Melo-Pfeifer, S. (2010). "O que diriam sobre os portugueses? ? ? ? [What would you say about Portuguese people?]: intercultural curiosity in multilingual chat-rooms". Language and Intercultural Communication, vol. 10, n 4. pp. 277-298.

Araújo e Sá, M. H., Hidalgo Downing, R., Melo-Pfeifer, S., Séré, A. \& Vela Delfa, C. (dir.). (2009). A intercompreensão em Línguas Românicas : conceitos, práticas, formação. Aveiero : Oficina Digital. Disponible en ligne : http://www.galanet.eu/publication/fichiers/

Araujo_e_Sa_et_al_Ed_2009.pdf

Bär, M. (2009). Förderung von Mehrsprachigkeit und Lernkompetenz : Fallstudien zu Interkomprehensionsunterricht mit Schülern der Klassen 8 bis 10. Giessener Beiträge zur Fremdsprachendidaktik. Tübingen : Narr.

Beacco, J.-C. \& Byram, M. (2005). "Préface". In Doyé : L'intercompréhension. Étude de référence. Guide pour l'élaboration des politiques linguistiques éducatives en Europe - De la diversité linguistique à l'éducation plurilingue. Strasbourg : Éditions du Conseil de l'Europe. Disponible en ligne : http:// www.coe.int/t/dg4/linguistic/Source/DoyeFR.pdf

Blanche-Benveniste. (1997). "Présentation". In Blanche-Benveniste, C. \& Valli, A. (dir.), L'intercompréhension : le cas des langues romanes - Le Français dans le monde. Recherches et applications. pp. 5-7.

Blanche-Benveniste, C. \& Valli, A. (dir.). (1997). L'intercompréhension : le cas des langues romanes - Le Français dans le monde. Recherches et applications.

Candelier, M., Camilleri-Grima, A., Castellotti, V., de Pietro, J.-F., Lörincz, I., Meißner, F.-J., Noguerol, A., et al. (2007). A travers les langues et les cultures. CARAP : cadre de références pour les approches plurielles des langues et des cultures. Graz: Centre européen pour les langues vivantes.

Capucho, F. (2008). "L'intercompréhension est-elle une mode ? Du linguiste citoyen au citoyen plurilingue". Pratiques, n 139/140. pp. 238-250.

Castagne, E. (2007a). "Formation initiale en français langue étrangère : actualité et perspectives". In Causa, M. (dir.). Formation initiale en français langue étrangère : actualité et perspectives - Le français dans le monde. Recherches et applications, $\mathrm{n}^{\circ}$ 41. pp. 66-75.

Castagne, E. (2007b). Les enjeux de l'intercompréhension. The stakes of intercomprehension. Reims: ÉPURE. Disponible en ligne : http://logatome.eu/Enjeux\%20intercomprehension.pdf

Chavagne, J.-P. (2010). "Apprendre à lire dans d'autres langues en pratiquant l'intercompréhension". Les Actes de Lecture, $\mathrm{n}^{\circ}$ 110. pp. 37-39. 
Chilmonczyk, R. (2002). "Zur Interkomprehension der deutschen und der nordischen Sprachen aus der Sicht polnischer Lerner". In Kischel, G. (dir.). Eurocom : mehrsprachiges Europa durch Interkomprehension in Sprachfamilien. Tagungsband des internationalen Fachkongresses im europäischen Jahr der Sprachen 2001, Hagen, 9. -10. November 2001, Hagen : Editiones EuroCom, Fernuniversität Hagen. pp. 367-384.

Dabène, L. (1995). "Apprendre à comprendre une langue voisine, quelles conceptions curriculaires ?" Études de linguistique appliquée, n 98. pp. 103-112.

Daff, M. \& Akissi Boutin, B. (2010). L'intercompréhension au cœur des processus d'apprentissage bilingue et tremplin pour une didactique plurilingue à visée convergente et intégrée en Afrique. Revue du Réseau des Observatoires du Français Contemporain en Afrique Noire, n 13. pp. 351-359.

Degache, C. (2003). "Présentation". Revue de linguistique et de didactique des langues, $\mathrm{n}^{\circ} 28$. Disponible en ligne $:$ http://lidil.revues.org/index1553.html?file=1

Degache, C. (2006). Didactique du plurilinguisme. Travaux sur l'intercompréhension et l'utilisation des technologies pour l'apprentissage des langues. Dossier présenté pour l'Habilitation à Diriger des Recherches. Synthèse de l'activité de recherche. UFR des sciences du langage - LIDILEM - Université StendhalGrenoble III. Disponible en ligne : http://www.galanet.be/publication/fichiers/ HDR2006_DegacheC.pdf

Degache, C. (2009). "Nouvelles perspectives pour l'intercompréhension (Afrique de l'ouest et Caraibe) et évolutions du concept". In Araújo e Sá, M. H., Hidalgo Downing, R., Melo-Pfeifer, S., Séré, A. \& Vela Delfa, C. (dir.). A intercompreensão em línguas românicas : conceitos, práticas, formação. Aveiero : Oficina Digital. pp. 81-102. Disponible en ligne : $\underline{\text { http://galanet.eu/publication/fichiers/ }}$ Araujo_e_Sa_et_al_Ed_2009.pdf

Délégation générale à la langue française et aux langues de France (DGLFLF) (dir.) (2006).

"L'intercompréhension entre langues apparentées". Disponible en ligne : http:// www.dglf.culture.gouv.fr/publications/intercomprehension.pdf

Doyé, P. (2005). L'intercompréhension. Guide pour l'élaboration des politiques linguistiques éducatives en Europe. De la diversité linguistique à l'éducation plurilingue. Étude de référence. Strasbourg : Conseil de l'Europe. Disponible en ligne : http://www.coe.int/t/dg4/linguistic/Source/DoyeFR.pdf

Escudé, P. (2010). "Origine et contexte d'apparition du terme d'intercompréhension dans sa première attestation (1913) chez le linguiste français Jules Ronjat (1864-1925)". RedinterIntercompreensão, $\mathrm{n}^{\circ} 1$. pp. 103-123.

European Commission. (n.d.). "L'intercompréhension, une approche différente à l'apprentissage des langues". Disponible en ligne : http://ec.europa.eu/languages/news/20100330-uneapproche-differente-a-l-apprentissage-des-langues_en.htm

Gajo, L. (2008). "L'intercompréhension entre didactique intégrée et enseignement bilingue". In Conti, V. \& Grin, F. (dir.), S'entendre entre langues voisines : vers l'intercomprehension Chêne-Bourg : Georg Editeur. pp. 131-150. Disponible en ligne : http://www.adeb.asso.fr/tours2007/ Gajo_Laurent_1.pdf

Gueidão, A., Melo-Pfeifer, S. \& Pinho, A. S. (2009). "Modelages d'intercompréhension - Que font les chercheurs du concept d'intercompréhension? Deux études de cas". In Araújo e Sá, M. H., Hidalgo Downing, R., Melo-Pfeifer, S., Séré, A. \& Vela Delfa, C. (dir.). A intercompreensão em línguas românicas : conceitos, práticas, formação. pp. 59-80.

Jamet, M.-C. (2010). "L'intercompréhension : de la définition d'un concept à la délimitation d'un champ de recherche ou vice versa ?" Publifarum - Autour de la définition, $\mathrm{n}^{\circ} 11$. Disponible en ligne : http://publifarum.farum.it/ezine_articles.php?art_id=144 
Jamet, M.-C. (2011). "L'intercompréhension, la clef d'un plurilinguisme rêvé et possible". Actes du IIème colloque de l'Alliance Française de São Paulo, São Paulo. Disponible en ligne : http:// www.aliancafrancesa.com.br/colloque2011/actes/Acte_Conference_MARIECHRISTINE_JAMET.pdf

Jamet, M.-C. \& Spita, D. (2010). "Points de vue sur l'intercompréhension : de définitions éclatées à la constitution d'un terme fédérateur". Redinter-Intercompreensão, $n^{\circ}$ 1. pp. 9-28.

Kerbrat-Orecchioni, C. (2002). L'énonciation. Paris : A. Colin.

Klein, H. G. \& Reissner, C. (2003). EuroComRom : historische Grundlagen der romanischen Interkomprehension. Aachen : Shaker, Editiones EuroCom.

Martins, F. R. de O. \& Bartolomeu, I. (2010). "Les représentations de l'intercompréhension à l'école primaire - quels défis pour la formation des enseignants ?" Actas de las XV Sesiones para docentes e Investigadores de Francés Lengua Extranjera. 1810-2010 : del francés del Iluminismo al francés de hoy. Rozário : Laborde Libros Editor.

Meißner, F.-J. (2003). "Esquisse d'une didactique de l'eurocompréhension. Tirage à part/preprint". In Meißner, F.-J., Meissner, C., Klein, H. G. \& Stegmann, T. D. (dir.). EuroComRom - les sept tamis. Lire les langues romanes dès le départ. Disponible en ligne $:$ http://www.silviaklein.de/ Europint/kurs/esquisse.pdf

Meißner, F.-J. (2004a). "Introduction à la didactique de l'eurocompréhension". In Meißner, F.-J., Meissner, C., Klein, H. G. \& Stegmann, T. D. (dir.). Les sept tamis : lire les langues romanes dès le départ. Avec une introduction à la didactique de l'eurocompréhension, Aachen : Shaker, Editiones EuroCom. pp. 7-140.

Meißner, F.-J. (2004b). "Modelling plurilingual processing and language growth between intercomprehensive languages. Towards the analysis of plurilingual language processing". In Zybatow, L. (dir.), Translation in der globalen Welt und neue Wege in der Sprach- und Übersetzerausbildung. Innsbrucker Ringvorlesung zur Translationswissenschaft II. Frankfurt am Main: Peter Lang. pp. 31-57. Disponible en ligne : http://archive.ecml.at/mtp2/Alc/pdf/ Meissner_2004.pdf

Meißner, F.-J. (2012). "REDINTER. Interkomprehension". In Fäcke, C., Martinez, H. \& Meißner, F.J. (dir.). Mehrsprachigkeit : Bildung - Kommunikation - Standards. 3. Bundeskongress des GMF. 16.-18. September 2010, Universität Augsburg. Stuttgart : Klett. pp. 234-238.

Meißner, F.-J., Beckmann, C. \& Schröder-Sura, A. (2009). Mehrsprachigkeit fördern, Vielfalt und Reichtum Europas in der Schule nutzen (MES): zwei deutsche Stichproben einer internationalen Studie in den Klassen 5 und $9 \mathrm{zu}$ Sprachen und Fremdsprachenunterricht. Tübingen : Narr. Disponible en ligne : http://www.uni-giessen.de/rom-didaktik/Multilingualism/download/facette1 deutschland.pdf

Melo, S. (2007). "O acto de solicitação e a co-construção da intercompreensão em interacções plurilingues : um estudo com chats romanófonos". In Capucho, M. F., Martins, A., Degache, C. \& Tost, T. (dir.). Diálogos em Intercompreensão. Actas do Colóquio, Lisboa, Setembro de 2007. Lisboa : Universidade Católica Editora. pp. 15-20. Disponible en ligne : http://redinter.eu/dialintercom/ Abstracts/Painel1/1.doc

Ollivier, C. (2010). "Représentations de l'intercompréhension chez les spécialistes du champ". REDINTER-Intercompreensão, $\mathrm{n}^{\circ}$ 1. pp. 47-69.

Ollivier, C. (2012). Grands témoins. In Degache, C. \& Garbarino, S. (dir.). Actes du colloque "Intercompréhension : compétences plurielles, corpus, intégration", Grenoble, 21-23 juin 2012. Disponible en ligne $:$ http://ic2012.u-grenoble3.fr/Contenu/Actes/gdtemoins.pdf 
Ollivier, C. \& Ferrão Tavares, C. (2010). O conceito de intercompreensão : origem, evolução e definições Redinter-Intercompreensão. Edições Cosmos.

Pinho, A. S. \& Andrade, A. I. (2008). "Programme de formation et parcours personnels d'apprentissage professionnel". Les Langues Modernes, $\mathrm{n}^{\circ}$ 1. pp. 53-61.

Ploquin, F. (2005). "Esprit de famille. On peut déjà se comprendre entre locuteurs de langues romanes". Le Monde diplomatique, janvier 2005. p. 23.

Ronjat, J. (1913). Essai de syntaxe des parlers provençaux modernes. Macon : Protat Frères.

Santos, L. (2007). Intercompreensão, aprendizagem de línguas e Didáctica do Plurilinguismo. Aveiro : Universidade de Aveiro. Disponible en ligne : http://ria.ua.pt/

bitstream/10773/1468/1/2008000918.pdf

Santos, L. \& Andrade, A. I. (2005). "Intercomprehension : developing student's ability to "dialogue" with languages". Educação em Línguas em contexto escolar : da intervenção à reflexão, Cadernos do LALE. Série Reflexões. pp. 581-585. Disponible en ligne : www.ua.pt/cidtff/lale/ Readobject.aspx ?obj $=14139$

Séré, A. (2009). "Une approche pragmatique du concept d'intercompréhension". In Araújo e Sá, M. H., Hidalgo Downing, R., Melo-Pfeifer, S., Séré, A. \& Vela Delfa, C. (dir.). A Intercompreensão em Línguas Românicas : conceitos, práticas, formação. Aveiro : Oficina Digital. pp. 33-44. Disponible en ligne $: \underline{\text { http://www.galanet.eu/publication/fichiers/Araujo_e_Sa_et_al_Ed_2009.pdf }}$

Shopov, T. (2009, December 2). "Definition of the concept Intercomprehension". E-Mail du $02 / 12 / 2009$.

Strasser, M. (2008). Verständigungsstrategien bei sehr geringen Sprachkenntnissen. Wien: Praesens.

Tafel, K. (2009). Slavische Interkomprehension. Eine Einführung. Tübingen: Narr.

Tost Planet, M. (2005). "I progetti europei d'intercomprensione tra parlanti di lingue romanze". In Benucci, A. (dir.), Le lingue romanze : una guida per l'intercomprensione. Torino : UTET libreria. pp. 15-54. Disponible en ligne : http://lingalog.net/dokuwiki/_media/intercomprehension/ rcslr/chapitre2_livre_benucci.pdf

Tost Planet, M. (2009). "Introduction". In Araújo e Sá, M. H., Hidalgo Downing, R., Melo-Pfeifer, S., Séré, A. \& Vela Delfa, C. (dir.). A Intercompreensão em Línguas Românicas : conceitos, práticas, formação . Galapro. pp. 19-29. Disponible en ligne : http://www.galanet.eu/publication/fichiers/ Araujo_e_Sa_et_al_Ed_2009.pdf

Tost Planet, M. (2010). Les approches plurielles : un nouveau paradigme pour l'enseignementapprentissage des langues. Expériences européennes et latino-américaines dans le domaine de l'intercompréhension". Synergies Chili, $\mathrm{n}^{\circ}$ 6. pp. 47-57.

Wuilmart, F. (2006). "La traduction littéraire : source d'enrichissement de la langue d'accueil". Revue de Littérature de l'Union Européenne, vol. 07, n 4. pp. 1-10.

Zeevaert, L. \& Möller, R. (2011). "Wege, Irrwege und Holzwege bei der Texterschließung. Empirische Untersuchungen zur germanischen Interkomprehension". In Meißner, F.-J., Capucho, F., Degache, C., Martins, A., Spita, D. \& Tost, M. (dir.). Intercomprehension. Learning, teaching, research. Akten des Europäischen Netzwerks Interkomprehension (REDINTER) im Rahmen des 3. Bundeskongresses des Gesamtverbandes Moderne Fremdsprachen, Universität Augsburg, 16.-18.9.2010. Tübingen: Narr, Giessener Beiträge zur Fremdsprachendidaktik. pp. 146-163. 


\section{Sites Internet}

Babelweb (2012). Consulté en septembre 2012 : http://www.babel-web.eu

EuroComGerm (nd). Consulté en septembre 2012 : http://www.eurocomcenter.eu/

EuroComRom (nd). Consulté en septembre 2012 : http://www.eurocomcenter.eu/

EuroComSlav (nd). Consulté en septembre 2012 : http://www.eurocomcenter.eu/

Euro-mania (nd). Consulté en septembre 2012 : http://www.euro-mania.eu/index.php

European Awareness and Intercomprehension. Consulté en septembre 2012 : http://eu-

intercomprehension.eu

Galanet (2012). Consulté en septembre 2012 : http://www.galanet.eu

Galapro (2012). Consulté en septembre 2012 : http://www.galapro.eu

Intercom (2009). Consulté en septembre 2012 : http://www.intercomprehension.eu

Intercompréhension européenne (ICE). Consulté en septembre 2012 : http://logatome.org/ice.htm

Intermar (2012). Consulté en septembre 2012 : http://www.intermar.ax/

Itinéraires romans (nd). Consulté en septembre 2012 : http://dpel.unilat.org/DPEL/Creation/IR/

index.fr.asp

Redinter (2011). Consulté en septembre 2012 : http://www.redinter.eu

\section{NOTES}

1. Pour plus de précisions sur le contexte d'apparition du concept et son évolution au fil des années, nous renvoyons aux articles de M.-C. Jamet (2010) et P. Escudé (2010).

2. Le Carap (Candelier et al., 2007) a effectué un travail important qui reste cependant, pensonsnous, à approfondir pour l'intercompréhension afin de définir, d'une part, s'il y a des compétences spécifiques à l'intercompréhension et, d'autre part, dans l'affirmative, ce qu'elles sont.

3. http://www.intercomprehension.eu

4. Soit dit en passant, on pourra s'étonner de ces singuliers chez des spécialistes du plurilinguisme.

\section{RÉSUMÉS}

Cela fera bientôt 20 ans que l'intercompréhension a émergé dans le domaine de la didactique des langues. De plus en plus de manifestations scientifiques et de publications traitent d'intercompréhension, un réseau européen réunit la plupart des spécialistes d'Europe, mais aussi d'autres continents. Pourtant, derrière ce mouvement de plus en plus unifié autour d'un concept relativement nouveau en didactique, une hétérogénéité des conceptions apparaît de plus en plus clairement. 
Cet article présente une analyse de 118 définitions publiées entre 1993 et 2012, il fait ressortir quelques tensions épistémologiques qui sous-tendent l'intercompréhension et propose une structuration du domaine.

Vor ca. 20 Jahren hielt das aus der Kontaktlinguistik stammende Konzept der Interkomprehension Einzug in die Sprachendidaktik. Immer mehr wissenschaftliche Veranstaltungen und Publikationen setzen sich mit Interkomprehension auseinander. Ein europäisches Netzwerk ist entstanden, das Experten aus Europa und anderen Kontinenten vereinigt. Jedoch lässt sich hinter der vordergründigen Einigkeit um diesen relativ jungen Begriff in der Didaktik eine Vielfalt an unterschiedlichen Auffassungen erkennen.

Dieser Artikel stellt eine Analyse von 118 Definitionen vor, die zwischen 1993 und 2012 publiziert wurden. Er zeigt einige epistemologische Spannungsfelder auf, die sich im Bereich der Interkomprehension auftun, und trägt zur Strukturierung des Forschungsfeldes bei.

\section{INDEX}

Schlüsselwörter : interkomprehension, epistemologie, mehrsprachige kommunikation, rezeption, interaktion

Mots-clés : Intercompréhension, épistémologie, communication plurilingue, réception, interaction

\section{AUTEUR}

\section{CHRISTIAN OLLIVIER}

Christian Ollivier est maître de conférences à l'université de La Réunion (département de FLE, laboratoire LCF). Ses domaines de spécialité sont la didactique des langues, l'approche (inter)actionnelle, l'utilisation d'Internet / du web social pour l'enseignement-apprentissage des langues et l'intercompréhension. Il a coordonné plusieurs groupes de recherche internationaux, dont celui du réseau européen de l'intercompréhension Redinter, de même que des projets nationaux et internationaux en lien avec ses thématiques, notamment le projet Babelweb (http:// www.babel-web.eu).

Courriel : ollivier.reunion@gmail.com Toile : http://www.christianollivier.eu

Adresse : Université de La Réunion, département de FLE, campus universitaire sud, 117, rue du Général Ailleret, 97430 Le Tampon. 